\title{
PRESENCIA DE PLAGUICIDAS ORGANOCLORADOS EN MUESTRAS BIOLÓGICAS DE TONINAS (Tursiops truncatus) Y MANATÍES (Trichechus manatus manatus) COLECTADAS EN EL SUR DEL GOLFO DE MÉXICO
}

\author{
Erik de Jesus FLORES SÁNCHEZ ${ }^{1}$, Marco Antonio GARCÍA-SALINAS ${ }^{1}$, \\ Alberto DELGADO-ESTRELLA ${ }^{1}$, Ana Laura CALDERÓN-GARCIDUEÑAS ${ }^{2}$, \\ Stefan M. WALISZEWSKI ${ }^{2}$, Raúl INFANZÓN-RUIZ ${ }^{2}$ y Rubén RUIZ-RAMOS ${ }^{2} *$
}

${ }^{1}$ Facultad de Ciencias Naturales, Universidad Autónoma del Carmen, Calle 56 №4 Esq. Av. Concordia, Col. Benito Juárez, 24180, Cd. del Carmen, Campeche, México

${ }^{2}$ Instituto de Medicina Forense, Universidad Veracruzana, S.S. Juan Pablo II esq. Reyes Heroles, Fracc. Costa Verde, Boca del Río, Veracruz, 94294, México

*Autor para correspondencia: ruruiz@uv.mx

(Recibido enero 2017; aceptado septiembre 2017)

Palabras clave: especies centinela, plaguicidas organoclorados, manatíes, toninas

\section{RESUMEN}

Los factores que impactan negativamente en el ambiente, invariablemente afectan la salud humana. Por ello, se requiere una oportuna detección y monitoreo de los contaminantes ambientales. El estudio de muestras biológicas, obtenidas de especies centinela, proporciona información valiosa sobre la presencia, la acumulación y los efectos de substancias tóxicas. Este trabajo registró las concentraciones de plaguicidas organoclorados (POC), determinadas en tejido graso de cinco organismos de la especie T. manatus manatus varados en distintas regiones de la costa del Golfo de México (Tabasco, Campeche y Quintana Roo), así como de 15 ejemplares de la especie T. truncatus que habitaron y vararon en el Área de Protección de Flora y Fauna "Laguna de Términos" en el Estado de Campeche. El rango de concentraciones encontradas en manatíes para DDT total ( $\sum$ DDT) fue $0.09-1.013 \mathrm{mg} / \mathrm{kg}$ en base lipídica, integrado por p,p'-DDE, o,p'-DDT y p,p'-DDT, mientras que para las toninas, fue de $0.334-10.757 \mathrm{mg} / \mathrm{kg}$. Los valores más altos de $\sum$ DDT en manatíes correspondieron a un espécimen proveniente de Quintana Roo. En toninas, el mayor nivel fue más de diez veces superior al valor máximo que se registró en manatíes. Aunque el tamaño de muestra es pequeño, los resultados sugieren que, la prohibición sobre el uso de estos compuestos ha tenido un impacto positivo en sus niveles en la zona muestreada, ya que las concentraciones de POC reportados en organismos de otras latitudes son superiores. Sin embargo, es necesario realizar más estudios, tanto en organismos vivos, como en los que varan y mueren en esta zona, para tener mayor certeza de las concentraciones actuales de POC en dichas poblaciones.

Key words: sentinel species, organochlorine pesticides, manatees, bottlenose dolphins 


\begin{abstract}
Factors that negatively impact the environment invariably affect human health. Therefore, timely detection and monitoring of environmental contaminants are required. The study of biological samples obtained from sentinel species provides valuable information about the presence, accumulation and effects of toxic substances. This work recorded the concentrations of organochlorine pesticides (OP) determined in blubber of five organisms of the species T. manatus manatus stranded in different regions of the coast of the Gulf of Mexico (Tabasco, Campeche and Quintana Roo) and in 15 specimens of the species T. truncatus that inhabited and were stranded in the Area of Protection of Flora and Fauna Lagoon of Terms in the State of Campeche. The range of concentrations found in manatees for total DDT ( $\Sigma$ DDT) was $0.09-1.013 \mathrm{mg} / \mathrm{kg}$ on lipid base, mainly composed of p,p'-DDE, o,p'-DDT and p,p'-DDT, while for the bottlenose dolphins was $0.334-10.757 \mathrm{mg} / \mathrm{kg}$. The highest values of $\sum$ DDT in manatees corresponded to a specimen from Quintana Roo. In bottlenose dolphins, the highest level was more than ten times higher than the maximum value registered in manatees. Although the sample size is small, the results suggest that the ban on the use of these compounds has had a positive impact on their levels in the sampled area, since the concentrations of OP reported in organisms from other latitudes are higher. However, it is necessary to carry out more studies, both in living organisms, as well as in those that are stranded and die in this area, in order to have greater certainty of the current concentrations of OP in these populations.
\end{abstract}

\section{INTRODUCCIÓN}

La degradación de los procesos ecológicos, la pérdida de especies y la contaminación de la trama de la vida, son factores que convergen y producen un impacto negativo en la salud ambiental de este planeta y, por lo tanto, en la calidad de vida y en la salud humana. Por lo anterior, la detección de agentes químicos contaminantes del ambiente y la evaluación de su impacto en éste, adquieren relevancia. El uso de muestras biológicas obtenidas de especies denominadas centinela resulta muy útil en la comprensión integral del problema (Aguirre y Tabor 2004, Moore 2008). Stahl (1997) define especie centinela, como cualquier organismo no humano que pueda reaccionar a un contaminante ambiental antes que éste impacte a los humanos, es decir, aquellas especies que nos pueden ayudar a detectar amenazas tempranas o niveles de toxicidad alarmantes provenientes del ambiente, que podrían poner en riesgo la salud humana. En términos generales, los organismos centinela, se agrupan en dos categorías: A) Los que presentan cierta sensibilidad a contaminantes químicos, toxinas biológicas o algunos patógenos, debido a su capacidad para incorporar estos elementos a través de la trama trófica; y B) Especies con fisiología o dieta similar a la de los humanos. A su vez, se considera que las especies "carismáticas", como los delfines, son buenos centinelas, debido a que tienen una atención pública especial y son más efectivos llevando el interés y la acción social a todo el ecosistema (Reddy et al. 2001).

En septiembre de1997 se reunieron en Frederick, Maryland, veintidós representantes del gobierno, de la academia y del sector privado para participar en un taller patrocinado por el Centro de Investigación de Salud Ambiental de las Fuerzas Armadas de los Estados Unidos (USACEHR), el Centro Nacional de Evaluación Ambiental de la Agencia de Protección del Ambiente de los Estados Unidos (EPA/NCEA) y la Agencia para el Registro de Substancias Tóxicas y Enfermedades (ATSDR). Este taller titulado "Using Sentinel Species Data to Address the Potential Human Health Effects of Chemicals in the Environment", se constituyó en un foro para explorar las cuestiones asociadas con la identificación de posibles efectos sobre la salud humana utilizando la información de especies centinela (Van Der Schalie et al. 1999)

El uso de diversas especies como sistemas de alerta temprana en evaluación del riesgo para la salud humana no es nuevo, ya que se tienen registros de su uso como organismos centinela desde comienzos del siglo XIX. A pesar de la falta de evidencias directas para demostrar que los productos químicos en el ambiente están afectando negativamente a los seres humanos, el potencial de estos compuestos para influir en aspectos como su reproducción y desarrollo ha sido claramente demostrado en estas poblaciones centinela (Van Der Schalie et al. 1999). Entre los impactos negativos al medio marino, que los humanos 
colectivamente han provocado, es posible percibir algunos síntomas en la salud ecológica, entre los que se pueden destacar, una mayor frecuencia e intensidad de la proliferación de algas nocivas y mayores tensiones ambientales en especies que ocupan los niveles tróficos superiores, esto último, como resultado de la sobrepesca, la pérdida de hábitats para crías y/o madres de éstas y la propagación de compuestos contaminantes persistentes como las dioxinas y los bifenilos policlorados (PCB) que se bioacumulan a través de la trama trófica (Aguirre y Tabor 2004). Estudios publicados desde hace más de cuatro décadas ya reportaban la presencia de anormalidades reproductivas en peces, aves y caimanes. Dichas alteraciones fueron atribuidas a la presencia en el ambiente de compuestos que actúan como disruptores endócrinos (Jackson y Halbert 1974, Colborn y Clement 1992, Rolland et al. 1997).

Actualmente existen distintos esfuerzos internacionales dirigidos a disminuir o eliminar el uso de estos compuestos. La Conferencia de las Naciones Unidas sobre el Medio Humano celebrada en 1972 en Estocolmo, fue la primera conferencia internacional sobre el ambiente que logró reunir a 113 naciones y otras partes interesadas, con el objeto de debatir cuestiones de preocupación común, lo que representó un verdadero parteaguas para el pensamiento moderno sobre ambiente y desarrollo. El Convenio de Estocolmo, suscrito el 21 de mayo del 2001 en Suecia, entró en vigor el 17 de mayo del 2004 y tuvo como objetivo principal "proteger la salud humana y ambiental de los compuestos orgánicos persistentes" (PNUMA 2003, PNUMA 2011). México, firmó este tratado el 23 de mayo del 2001 y fue ratificado por el Congreso de la Unión el día 10 de febrero del 2003, con lo que nuestro país se constituyó en el primer país en Latinoamérica en ratificarlo (SEMARNAT 2013). A nivel regional México también es signatario del Acuerdo de Cooperación Ambiental de América del Norte 1993. En este contexto, el presente trabajo evaluó los niveles de plaguicidas organoclorados en cinco muestras de grasa corporal de manatíes (Trichechus manatus manatus), provenientes de las regiones donde se han obtenido registros directos e indirectos de la presencia del manatí en los últimos 20 años (Fig. 1), el Parque eco-arqueológico Xcaret, Quintana Roo, la Universidad Juárez Autónoma de Tabasco y los varamientos ocurridos en tres regiones de Estado de Campeche: 1) Río San Pedro, localidad de Nuevo Campechito; 2) Atasta; y 3) Ciudad del

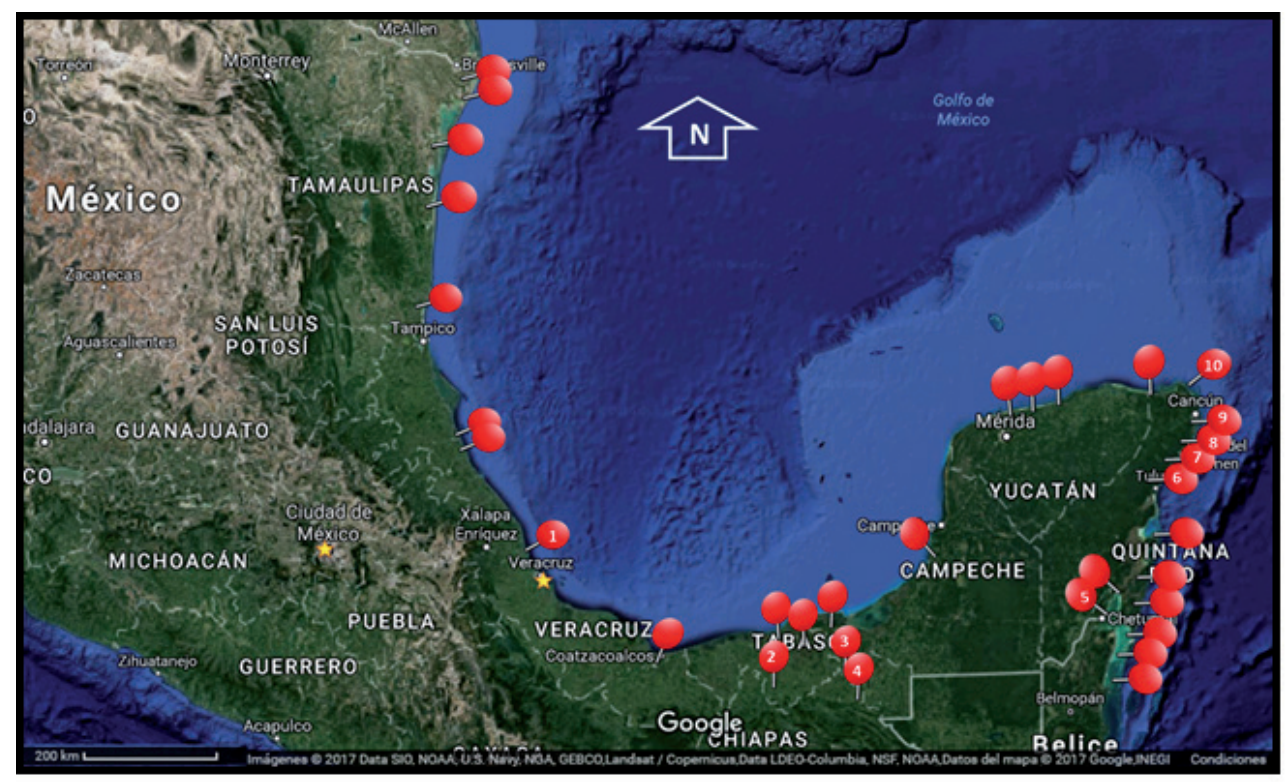

Fig. 1. Distribución actual del manatí T. manatus manatus en el Golfo de México. Las áreas señaladas indican las regiones donde se han obtenido registros directos e indirectos de la presencia del manatí en los últimos 20 años (aunque en la zona centro y norte de Tamaulipas y zona norte de Yucatán los reportes actuales han sido muy esporádicos). Los señaladores numerados indican los sitios donde se mantienen manatíes en cautiverio y semicautiverio 1) Acuario de Veracruz, 2) Yumka y UJAT [Villahermosa], 3) Jonuta, 4) Ecoparque Aluxes, Palenque, 5) Chetumal, 6) Xel-há, 7) Puerto Aventuras, 8) Xcaret, 9) Cozumel y 10) Isla Mujeres. Actualizado de OrtegaArgueta y Morales-Vela (2005) (Tomada y modificada de Google maps) 
Carmen. Así mismo, se evaluaron muestras de 15 toninas (Tursiops truncatus) que son habitantes regulares y que vararon en el Área de Protección de Flora y Fauna "Laguna de Términos" (APFFLT) (Fig. 2), considerada la zona de crianza más importante en el Golfo de México para esta especie (Delgado-Estrella 2002). La importancia de este estudio radica en la comparación de los niveles y los tipos de compuestos organoclorados encontrados en tejido graso de manatíes y de toninas, ya que de éstos últimos, una parte de la población se encuentran en la zona y es considerada residente, por lo que están expuestos durante todo su ciclo vida a estos contaminantes. Con base en los resultados, se discute la relación entre las cantidades de plaguicidas organoclorados detectadas y las medidas regulatorias establecidas para estas substancias; de esta manera se analiza el éxito de estas últimas en la restauración ecológica pasiva en la región.

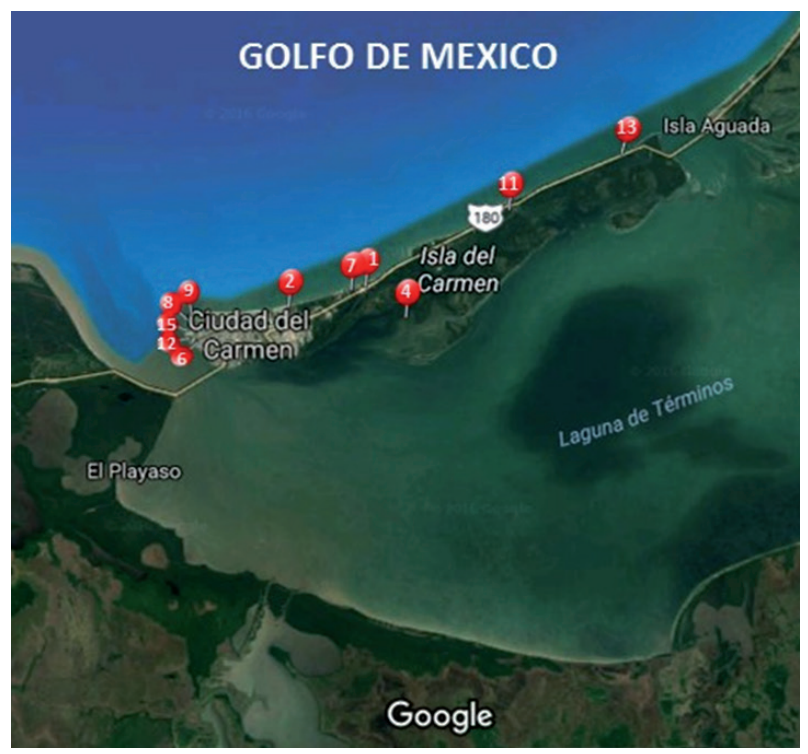

Fig. 2. Distribución espacial de varamientos de T. truncatus en la Isla del Carmen, Campeche. Las áreas señaladas con números indican las regiones del Área de Protección de Flora y Fauna Laguna de Términos, donde ocurrieron los varamientos de toninas de las cuales fueron obtenidas las muestras de tejido graso que se analizaron para evaluar presencia de plaguicidas organoclorados. (Tomada y modificada de Google maps)

\section{MATERIALES Y MÉTODOS}

\section{Colecta de muestras}

Se colectaron muestras de grasa de 15 delfines muertos, arribados a la playa de la Isla del Carmen, Campeche, México y áreas adyacentes, (Fig. 2), entre marzo de 2013 y enero de 2016, siguiendo el
Protocolo de Atención para Varamiento de Mamíferos Marinos (SEMARNAT 2014). En dicho protocolo se establecen distintas categorías de acuerdo con el estado de descomposición de los animales. Se realizó un corte de piel y grasa de aproximadamente $5 \times 5 \mathrm{~cm}$ en la región dorso-lateral y las piezas se mantuvieron en congelación a $-20^{\circ} \mathrm{C}$ hasta el momento del análisis. Para el estudio únicamente se consideraron las muestras de ejemplares que de acuerdo con el protocolo se encuentran entre los estados de descomposición "c, d y e". Mismos que se mencionan a continuación:

c) Animales muertos sin presentar señas de descomposición: aquellos que mueren durante la atención del varamiento o varan a pocas horas de haber fallecido en el mar.

d) Animales que presentan los primeros signos de descomposición: presentan ligera hinchazón del vientre y opacidad en los ojos.

e) Animales en descomposición: presentan gran hinchazón, la lengua y ojos están fuera, se observa sangre en los orificios, la piel empieza a perderse y se observa grasa en la superficie del cuerpo.

Para el caso de los manatíes, cinco bloques de piel y grasa de organismos varados fueron colectados por diferentes centros de investigación a lo largo de la costa del Golfo de México (Fig. 1), entre los años 2011-2015. El material biológico se mantuvo en congelación a $-20^{\circ} \mathrm{C}$.

Muestra 1. Obtenida por la Universidad Juárez Autónoma de Tabasco, Proyecto Manatí-UJAT. Varamiento de un macho adulto de aproximadamente 2.8 metros de longitud, ocurrido el 19 de diciembre del 2014 en la Laguna de Las Ilusiones, Tabasco.

Muestra 2. Obtenida del varamiento de un macho de 2.64 metros y aproximadamente 400 kilogramos, ocurrido el 28 de agosto del 2013 en el Rio San Pedro, localidad de Nuevo Campechito, situado en el municipio de Ciudad del Carmen, Campeche.

Muestra 3. Obtenida del varamiento de un macho de aproximadamente 2.57 metros de longitud y un peso aproximado de 300 kilogramos. Se encontró muerto en la Península de Atasta, Campeche el 5 de julio del 2011.

Muestra 4. Obtenida del varamiento de un macho de aproximadamente 2.69 metros de longitud, ocurrido el 26 de septiembre del 2011 frente a la Tercera Región Naval en Ciudad del Carmen, Campeche.

Muestra 5. Obtenida de un ejemplar macho en el Parque eco-arqueológico Xcaret, Quintana Roo, el 11 de junio del 2014. 


\section{Análisis de las muestras}

Para el análisis cualitativo y cuantitativo de las muestras de grasa colectadas, se utilizó un cromatógrafo de gases marca Varian modelo $3400 \mathrm{CX}$ con detector de captura de electrones y una columna capilar megaboro SPB-608 de $30 \mathrm{~m}$ de longitud y $320 \mu \mathrm{m}$ (i.d.). Las condiciones de trabajo fueron: columna en un programa de temperatura de $193^{\circ} \mathrm{C}$ (7 min), aumentándola a $6{ }^{\circ} \mathrm{C} / \mathrm{min}$ hasta llegar a $250^{\circ} \mathrm{C}$, que se mantuvo durante $20 \mathrm{~min}$. El gas de arrastre fue nitrógeno con flujo $6.3 \mathrm{~mL} / \mathrm{min}$ y se inyectó $1 \mu \mathrm{L}$ del extracto en modo "splitless". La determinación cualitativa y cuantitativa por cromatografía de gases se realizó por medio del paquete Workstation versión 6.0 comparando los tiempos de retención y calculando las áreas bajo los picos correspondientes de cada muestra y de los estándares de $\mathrm{HCB}, \alpha-\mathrm{HCH}$, $\beta-\mathrm{HCH}, \gamma-\mathrm{HCH}, \mathrm{p}, \mathrm{p}$ '-DDE, o,p'-DDT, p,p'-DDT con concentraciones de $0.1 \mathrm{mg} / \mathrm{L}$ de disolvente. Para la purificación de los extractos, se utilizó $\mathrm{H}_{2} \mathrm{SO}_{4}$ para precipitar las grasas e hidrolizar el complejo de plaguicidas con los compuestos endógenos de los tejidos (Waliszewski et al. 2003).

La extracción para la determinación de analitos y la purificación de los extractos, se hicieron de acuerdo con el método de Waliszewski y cols. (1982, 2003). $\mathrm{La}$ grasa fue descongelada y macerada con $\mathrm{Na}_{2} \mathrm{SO}_{4}$ hasta obtener un polvo seco y homogéneo. Cada producto obtenido fue colocado en una columna de vidrio para la extracción de los compuestos lipídicos que contienen los plaguicidas organoclorados, con $100 \mathrm{~mL}$ de hexano. Se tomaron $10 \mathrm{~mL}$ del extracto y se colocaron en un roto-vapor a $40^{\circ} \mathrm{C}$ para volatilizar el disolvente a sequedad y obtener por gravimetría la concentración total de las grasas, que constituyen el peso de la muestra $(\mathrm{mg})$. Se tomaron $10 \mathrm{~mL}$ adicionales del extracto y se colocaron en un tubo de ensaye con tapa de rosca, se agregó $1 \mathrm{~mL}$ de $\mathrm{H}_{2} \mathrm{SO}_{4}$ y la mezcla se agitó vigorosamente por tres minutos. El sobrenadante se filtró en una columna con $\mathrm{Na}_{2} \mathrm{SO}_{4}$ y se recuperó el extracto enjuagándolo con hexano. El extracto con el enjuague recuperado, se concentró en el rota-vapor a $40^{\circ} \mathrm{C}$ hasta unas gotas, las cuales se trasvasaron a un vial, aforándolo a $1.0 \mathrm{~mL}$. Se inyectó un volumen de $1 \mu \mathrm{L}$ del extracto en el cromatógrafo de gases.

Para establecer la calidad analítica, se realizó el estudio de adición de una mezcla de estándares a una porción homogénea de grasa de pollo que contenía residuos mínimos de plaguicidas organoclorados, menores al límite de detección $(0.001 \mathrm{mg} / \mathrm{kg}$ de grasa extraída). A ésta se le agregó $1.0 \mathrm{~mL}$ de la solución hexánica de los plaguicidas organoclorados con las concentraciones de 0.01-0.02 mg/L dependiendo del plaguicida y que corresponden al valor 10 veces superior al límite señalado. Después de la fortificación, se realizó todo el proceso analítico. Los resultados del estudio de recuperación, realizado en 10 repeticiones, mostraron valores entre 89 a $98 \%$ dependiendo del plaguicida estudiado; el porcentaje de varianza fue menor al $10 \%$, siendo éste adecuado para el estudio.

\section{RESULTADOS}

Se analizaron en total cinco muestras de manatíes machos provenientes de Tabasco (1), Campeche (3: Nuevo Campechito, Atasta y Cd. del Carmen) y Quintana Roo (1), así como 15 de toninas provenientes de la región de la Laguna de Términos, Campeche. Los resultados fueron los siguientes:

\section{Hexaclorobenceno (HCB)}

Los niveles mayores de HCB fueron encontrados en las muestras biológicas obtenidas de manatíes provenientes de Quintana Roo $(0.062 \mathrm{mg} / \mathrm{kg})$ y de Nuevo Campechito $(0.041 \mathrm{mg} / \mathrm{kg})$. En los ejemplares obtenidos de Tabasco, Atasta y Cd. del Carmen no se registraron niveles de este compuesto (Cuadro I). En contraste, en los tejidos grasos de toninas provenientes de Laguna de Términos se registró el nivel más elevado en el espécimen $7(0.163 \mathrm{mg} / \mathrm{kg})$, en doce muestras se determinaron concentraciones en un rango desde 0.030 a $0.089 \mathrm{mg} / \mathrm{kg}$ y solo los ejemplares 4 y 8 no mostraron concentraciones por encima del límite de detección $(0.001 \mathrm{mg} / \mathrm{kg}$ ) (Cuadro II).

\section{Alfa-hexaclorociclohexano ( $\alpha-\mathrm{HCH})$}

Para las muestras obtenidas de manatíes, éste isómero estuvo presente únicamente en las provenientes de Nuevo Campechito $(0.027 \mathrm{mg} / \mathrm{kg})$ y de Tabasco $(0.008 \mathrm{mg} / \mathrm{kg})$. No se observaron niveles detectables $(>0.001 \mathrm{mg} / \mathrm{kg}$ ) en las obtenidas en Atasta, Cd. del Carmen y Quintana Roo (Cuadro I). Sin embargo, para las determinaciones en toninas de Laguna de Términos los niveles más altos de éste isómero fueron en las muestras 1 y 7 , con valores de 0.040 y 0.057 $\mathrm{mg} / \mathrm{kg}$, respectivamente, mientras que el nivel más bajo se presentó en la muestra 3, con $0.007 \mathrm{mg} / \mathrm{kg}$. En las doce determinaciones restantes se detectaron niveles entre $0.010-0.036 \mathrm{mg} / \mathrm{kg}$ (Cuadro II).

\section{Beta-hexaclorociclohexano ( $\beta-\mathrm{HCH})$}

Las mayores concentraciones de $\beta-\mathrm{HCH}$ se detectaron en el manatí proveniente de Quintana Roo $(0.078 \mathrm{mg} / \mathrm{kg})$, mientras que las concentraciones notoriamente menores, se encontraron en las muestras 
CUADRO I. CONCENTRACIÓN (mg/kg EN BASE LIPÍDICA) DE PLAGUICIDAS EN MUESTRAS DE TEJIDO GRASO DE MANATÍES (T. manatus manatus)

\begin{tabular}{|c|c|c|c|c|c|c|c|c|}
\hline \multirow{2}{*}{ Muestras } & \multicolumn{8}{|c|}{ Plaguicida } \\
\hline & $\mathrm{HCB}$ & $\alpha-\mathrm{HCH}$ & $\beta-\mathrm{HCH}$ & $\gamma-\mathrm{HCH}$ & DDE & $\mathrm{o}, \mathrm{p}$-DDT & $\mathrm{p}, \mathrm{p}$ '-DDT & S DDT \\
\hline 1 ( Tabasco) & ND & 0.008 & 0.019 & 0.022 & 0.096 & 0.072 & 0.053 & 0.221 \\
\hline 2 (Nvo. Campechito) & 0.041 & 0.027 & ND & ND & ND & 0.090 & ND & 0.090 \\
\hline 3 (Atasta, Campeche) & ND & ND & ND & ND & ND & ND & ND & ND \\
\hline 4 (Cd. del Carmen $)$ & ND & ND & 0.006 & ND & 0.044 & 0.203 & 0.089 & 0.336 \\
\hline 5 (Quintana Roo) & 0.062 & ND & 0.078 & ND & 0.318 & 0.695 & ND & 1.013 \\
\hline
\end{tabular}

LD: $0.001 \mathrm{mg} / \mathrm{Kg}$ (miligramo de plaguicidas/Kg de grasa extraída)

ND: No detectado

CUADRO II. CONCENTRACIÓN (mg/kg en base lipídica) DE PLAGUICIDAS EN MUESTRAS DE TEJIDO GRASO DE TONINAS (T. truncatus)

\begin{tabular}{cccccccccc}
\hline Muestras & \multicolumn{9}{c}{ Plaguicida } \\
\cline { 2 - 9 } & HCB & $\alpha$-HCH & $\beta$-HCH & $\gamma$-HCH & p, p'-DDE & o, p-DDT & p, p'-DDT & $\sum$-DDT \\
\hline 1 & 0.030 & 0.040 & ND & ND & 10.037 & 0.113 & 0.607 & 10.757 \\
2 & 0.043 & 0.010 & ND & ND & 0.294 & 0.070 & 0.204 & 0.568 \\
3 & 0.030 & 0.007 & ND & ND & 0.361 & 0.051 & 0.072 & 0.484 \\
4 & ND & 0.036 & ND & 0.041 & 0.498 & 0.136 & 0.439 & 1.073 \\
5 & 0.086 & 0.026 & ND & ND & 0.254 & 0.047 & 0.033 & 0.334 \\
6 & 0.089 & 0.030 & ND & ND & 0.499 & 0.212 & 0.243 & 0.954 \\
7 & 0.163 & 0.057 & ND & 0.103 & 1.278 & 0.410 & 0.555 & 2.243 \\
8 & ND & 0.022 & ND & ND & 0.037 & 0.026 & 0.063 & 0.126 \\
9 & 0.051 & 0.013 & 0.006 & 0.015 & 0.130 & 0.040 & 0.046 & 0.216 \\
10 & 0.062 & 0.017 & ND & ND & 8.559 & 0.230 & 0.481 & 9.270 \\
11 & 0.075 & 0.018 & ND & ND & 0.623 & 0.119 & 0.324 & 1.066 \\
12 & 0.051 & 0.013 & ND & ND & 1.438 & 0.097 & 0.351 & 1.886 \\
13 & 0.071 & 0.032 & ND & ND & 0.194 & 0.088 & 0.090 & 0.372 \\
14 & 0.079 & 0.027 & ND & 0.040 & 7.656 & 0.263 & 0.445 & 8.364 \\
15 & 0.074 & 0.019 & ND & ND & 0.249 & 0.060 & 0.106 & 0.415 \\
\hline
\end{tabular}

LD: $0.001 \mathrm{mg} / \mathrm{Kg}$ (miligramo de plaguicidas $/ \mathrm{Kg}$ de grasa extraída) ND: No detectado

de Tabasco $(0.019 \mathrm{mg} / \mathrm{kg})$ y de Cd. del Carmen $(0.006 \mathrm{mg} / \mathrm{kg})$. No se registraron niveles detectables $(>0.001 \mathrm{mg} / \mathrm{kg})$ en los ejemplares de Nuevo Campechito y Atasta (Cuadro I). En las toninas, éste isómero se detectó únicamente en la muestra 9 en una concentración de $0.006 \mathrm{mg} / \mathrm{kg}$ (Cuadro II).

\section{Gama-hexachlorociclohexano ( $\gamma$-HCH) (Lindano)}

En manatíes, el valor más alto de Lindano se encontró en el proveniente de Tabasco $(0.022 \mathrm{mg} / \mathrm{kg})$. No se registraron niveles detectables en los otros ejemplares (Cuadro I). En toninas las muestras 4, 7, 9 y 14 presentaron $0.041,0.103,0.015$ y $0.040 \mathrm{mg} / \mathrm{kg}$ de este plaguicida respectivamente, en el resto no se detectó este compuesto (Cuadro II).

\section{para, para'-diclorodifenildicloroetileno (p,p'- DDE)}

Los valores más altos de p,p'-DDE se observaron en manatíes provenientes de Quintana Roo $(0.318 \mathrm{mg} / \mathrm{kg})$ seguido de Tabasco $(0.096 \mathrm{mg} / \mathrm{kg})$ y Cd. del Carmen $(0.044 \mathrm{mg} / \mathrm{kg})$. No se registraron niveles detectables para Nuevo Campechito y Atasta (Cuadro I). Para el caso de los ejemplares de toninas provenientes de Laguna de Términos, en todos se manifestó la presencia de p,p'-DDE, en un rango de $0.037 \mathrm{mg} / \mathrm{kg}$ (muestra 8), hasta $10.037 \mathrm{mg} / \mathrm{kg}$ (muestra 1). Además, los niveles más altos correspondieron a los especímenes 1,10 y 14, con concentraciones de $10.037,8.559$ y $7.656 \mathrm{mg} / \mathrm{kg}$, respectivamente (Cuadro II). 
orto, para-diclorodifeniltricloroetano (o,p'-DDT)

La concentración más altas de o,p'-DDT en manatíes, fue del organismo proveniente de Quintana Roo $(0.695 \mathrm{mg} / \mathrm{kg})$, seguida por los de Cd. del Carmen $(0.203$ $\mathrm{mg} / \mathrm{kg})$, Nuevo Campechito $(0.090 \mathrm{mg} / \mathrm{kg}$ ) y Tabasco $(0.072 \mathrm{mg} / \mathrm{kg})$. Mientras que no se registró este compuesto en el organismo varado en Atasta (Cuadro I). En todas las toninas, se revelaron niveles cuantificables de este compuesto, en un rango desde 0.026 (muestra 8) hasta $0.410 \mathrm{mg} / \mathrm{kg}$ (muestra 7) (Cuadro II).

para, para'-diclorodifeniltricloroetano (p,p'DDT)

Las cantidades más altas determinadas de p,p'DDT en manatíes, correspondieron a los provenientes de Cd. del Carmen $(0.089 \mathrm{mg} / \mathrm{kg})$ y de Tabasco $(0.053$ $\mathrm{mg} / \mathrm{kg}$ ). (Cuadro I). En las muestras de toninas la mayor concentración observada de p,p'-DDT fue de $0.607 \mathrm{mg} / \mathrm{kg}$ (organismo 1), mientras que la menor concentración fue de $0.033 \mathrm{mg} / \mathrm{kg}$ (organismo 5) (Cuadro II).

Diclorodifeniltricloroetano total (p,p'DDT + o,p'DDT + p,p'DDE = $\sum$ DDT).

La evaluación, considerando S DDT, mostró la concentración más alta para manatíes en Quintana Roo $(1.013 \mathrm{mg} / \mathrm{kg})$, seguida por el proveniente de $\mathrm{Cd}$. del Carmen $(0.336 \mathrm{mg} / \mathrm{kg})$ y de Tabasco $(0.221 \mathrm{mg} / \mathrm{kg})$ (Cuadro I). La menor concentración se obtuvo en la grasa colectada en Nuevo Campechito $(0.090 \mathrm{mg} / \mathrm{kg})$. En los tejidos obtenidos de toninas, las concentraciones más altas correspondieron a tres de los 15 colectados $(1,10$ y 14$)$ con valores de $10.757,9.270$ y $8.364 \mathrm{mg} / \mathrm{kg}$ respectivamente, seguidas por uno con $2.243 \mathrm{mg} / \mathrm{kg}$ (7) y los 11 restantes tuvieron concentraciones en un rango desde 0.126 a $1.886 \mathrm{mg} / \mathrm{kg}$ (Cuadro II).

\section{DISCUSIÓN}

Son escasas las investigaciones realizadas en México para conocer la presencia de tóxicos ambientales, tales como plaguicidas organoclorados, en mamíferos acuáticos, como sirenios (manatíes y dugongos), cetáceos (ballenas, delfines y marsopas), pinnípedos (focas verdaderas, otáridos y morsas), entre otros. Por ende, se conoce poco sobre los niveles originados por la acumulación ambiental, patologías asociadas y efectos tóxicos en general. El presente estudio aporta evidencia de un monitoreo de compuestos organoclorados en tejido graso de diferentes especímenes de T. manatus manatus y de
T. truncatus varados en diversas regiones del Golfo de México. Se considera que la presencia de estos compuestos se debe al hecho de que en las regiones donde se realizaron las colectas (Tabasco, Campeche y Quintana Roo), donde habitan los manatíes, así como donde residen y varan las toninas (Área de Protección de Flora y Fauna "Laguna de Términos") se llevan a cabo actividades antrópicas, en las cuales se hace uso de agroquímicos. Los resultados de la muestra proveniente de Laguna de las Ilusiones, en el Estado de Tabasco, evidencian la presencia de $\mathrm{HCH}$ y sus isómeros $\alpha-\mathrm{HCH}, \beta-\mathrm{HCH}$ y $\gamma-\mathrm{HCH}$ $\mathrm{y}$ en concentraciones mayores, formas isoméricas de DDT (o,p'-DDT y p,p'-DDT) y su metabolito p,p'-DDE. En la grasa obtenida de un manatí varado en Nuevo Campechito, la concentración de los isómeros o,p'-DDT $(0.090 \mathrm{mg} / \mathrm{kg})$ y $\alpha-\mathrm{HCH}$ $(0.027 \mathrm{mg} / \mathrm{kg})$, fue mayor en comparación a la observada en Tabasco. Adicionalmente, se observó también HCB en el ejemplar proveniente de Quintana Roo. No obstante, en este último también se cuantificaron los isómeros $\beta$-HCH, o,p'-DDT y el metabolito p,p'-DDE con las concentraciones más altas $(0.062,0.078,0.318,0.695 \mathrm{mg} / \mathrm{kg}$ respectivamente). En el animal varado en $\mathrm{Cd}$. del Carmen se observaron los niveles más bajos de $\beta-\mathrm{HCH}$ y de p,p'-DDE (0.006 y $0.044 \mathrm{mg} / \mathrm{kg}$ respectivamente), aunque también fue revelada la presencia del isómero o,p'-DDT $(0.203 \mathrm{mg} / \mathrm{kg})$ con la segunda concentración más alta y el isómero p,p'-DDT con la concentración más alta analizada $(0.089 \mathrm{mg} / \mathrm{kg})$. Para la muestra de tejido proveniente de la Laguna de Atasta, no se encontró ninguno de los compuestos anteriores. En contraste, en trece de las quince toninas analizadas, el HCB estuvo presente en un rango de concentraciones de $0.030-0.163 \mathrm{mg} / \mathrm{kg}$, siendo la concentración más alta 2.6 veces mayor que la más alta registrada en manatíes provenientes de Quintana Roo. En todas las muestras se observó la presencia del isómero $\alpha-\mathrm{HCH}$ en un intervalo de 0.007-0.057 $\mathrm{mg} / \mathrm{kg}$, es decir, la concentración menor fue similar a la más baja detectada en los manatíes $(0.008 \mathrm{mg} / \mathrm{kg})$ (Tabasco) (Cuadro I), pero la concentración mayor, del mismo intervalo, fue más de dos veces la concentración más alta en manatíes $(0.027 \mathrm{mg} / \mathrm{kg})$ (Nuevo Campechito) (Cuadro I). El isómero $\beta$-HCH solo se encontró en la muestra 9 de toninas, en niveles similares a la menor concentración reportada en el manatí procedente de $\mathrm{Cd}$. del Carmen, mientras que el isómero $\gamma$-HCH solo se determinó en cuatro ejemplares, en un rango de $0.015-0.103 \mathrm{mg} / \mathrm{kg}$, siendo la concentración más alta casi 4.7 veces mayor a la determinada en la única muestra de manatí 
CUADRO III. COMPARACIÓN DE NIVELES (mg/kg en base lipídica) DE PLAGUICIDAS ORGANOCLORADOS REPORTADOS EN MUESTRAS DE T. truncatus EN OTROS ESTUDIOS

\begin{tabular}{|c|c|c|c|c|c|c|}
\hline \multirow[t]{2}{*}{ Área de estudio } & \multirow[t]{2}{*}{ Año/s } & \multirow[t]{2}{*}{ Sexo } & \multicolumn{3}{|c|}{$\sum \mathrm{DDT}$} & \multirow[t]{2}{*}{ Referencias } \\
\hline & & & $\bar{X}$ & Max & Min & \\
\hline USA & $1983-1985$ & $\begin{array}{l}8 \mathrm{M} \\
7 \mathrm{H}\end{array}$ & $\begin{array}{c}31.8 \\
4.62\end{array}$ & $\begin{array}{r}1115 \\
8.02\end{array}$ & $\begin{array}{l}78 \\
0.69\end{array}$ & King (1987) \\
\hline USA & 1990 & $\begin{array}{l}6 \mathrm{M} \\
10 \mathrm{H}\end{array}$ & $\begin{array}{l}38 \\
3.7\end{array}$ & $\begin{array}{l}78 \\
14\end{array}$ & $\begin{array}{l}15 \\
0.6\end{array}$ & Kuehl y Haebler (1995) \\
\hline Italia & $1987 / 1992$ & $\begin{array}{l}5 \mathrm{M} \\
2 \mathrm{H}\end{array}$ & $\begin{array}{l}15 \\
3.98\end{array}$ & $\begin{array}{r}57.68 \\
4.66\end{array}$ & $\begin{array}{l}0.64 \\
3.30\end{array}$ & Marsili y Focardi (1997) \\
\hline Italia & 1992 & $\begin{array}{l}5 \mathrm{M} \\
2 \mathrm{H}\end{array}$ & $\begin{array}{l}399.6 \\
140\end{array}$ & $\begin{array}{r}1100 \\
260\end{array}$ & $\begin{array}{l}48 \\
20\end{array}$ & Corsolini et al. (1995) \\
\hline USA & 1995 & 33 ambos & 15.3 & 74.6 & 0.43 & Salata et al. (1995) \\
\hline USA & $1997-2002$ & 73 ambos & 5.19 & 8.72 & 1.13 & Hansen et al. (2004) \\
\hline USA & $2003-2005$ & $40 \mathrm{M}$ & 26.2 & 86.8 & 9.55 & Adams et al. (2014) \\
\hline USA & 2004-2006 & 173 ambos & 7.96 & 33.1 & 0.482 & Wilson et al. (2012) \\
\hline México & $2013-2016$ & 15 ambos & 2.54 & 10.75 & 0.126 & Este trabajo \\
\hline
\end{tabular}

(2) en que se encontró este compuesto proveniente de Tabasco. En todos los casos se detectaron metabolitos e isómeros de DDT y los valores más altos de $\sum$ DDT fueron superiores al valor máximo cuantificado en grasa de manatíes, por más de diez veces (muestra 6, proveniente de Quintana Roo) (Cuadro I). Sin embargo, esta concentración máxima, es menor a la registrada en la misma especie en otros estudios realizados en diferentes países, considerando ambos sexos (Cuadro III). Aguilar et al. (2002) establecieron, basados en la información de múltiples estudios realizados con varias especies de mamíferos marinos, cinco valores medios de concentraciones de DDT de distribución geográfica por especie. La concentración máxima de $\sum$ DDT observada en el presente estudio (10.757 $\mathrm{mg} / \mathrm{kg}$ ), está ligeramente por arriba del valor medio inferior, del intervalo de concentraciones establecida para T. truncatus en el Golfo de México (10-30 $\mathrm{mg} / \mathrm{kg}$ ). Dichas concentraciones podrían considerarse "seguras" al compararlas con las reportadas en otros mamíferos acuáticos, en otras regiones del mundo, ya que se han reportado concentraciones de plaguicidas organoclorados en otros sirenios como los Dugongos (Dugong dugon) a lo largo de Queensland, Australia, en un rango de $0.0012-0.066 \mathrm{mg} / \mathrm{kg}$ de p,p'-DDT y 0.0009-0.161 mg/kg para p,p'-DDE (Vetter et al. 2001, Haynes et al. 2005). Por su parte,
Brown (1978) también reportó concentraciones de plaguicidas organoclorados en grasa subcutánea $>0.9 \mathrm{mg} / \mathrm{kg}$ para lobos marinos de la zona de California, mientras que para ballenas grises, que se alimentan con anfípodos en el Mar de Bering, se han registrado concentraciones de DDT de $2.3 \mathrm{mg} / \mathrm{kg}$. Wagemann y Muir (1984) determinaron que concentraciones de $50 \mathrm{mg} / \mathrm{kg}$ de DDT, se asociaron con alteraciones reproductivas y patológicas en cetáceos. Sin embargo, Lahvis et al. (1995) reportaron que concentraciones mucho menores de $\mathrm{p}, \mathrm{p}$ '-DDT $(\leq 0.00003 \mathrm{mg} / \mathrm{kg})$, así como de p,p'-DDE $(\leq 0.0005$ $\mathrm{mg} / \mathrm{kg}$ ) fueron capaces de producir alteraciones en la capacidad proliferativa de linfocitos aislados de sangre periférica de T. truncatus, en presencia de concanavalina A (Con A) o fitohemaglutinina (PHA).

En los Estados de Tabasco y Campeche los cultivos de caña, frijol, maíz, sandía, arroz, sorgo y hortalizas son importantes para la economía local, por lo que también se ha favorecido el uso de herbicidas, fungicidas, así como de insecticidas para el control de vectores ( Benítez y Bárcenas 1996, Albert y Loera Gallardo 2005). Adicionalmente, Rendón-von Osten et al. (2005) reportaron, la presencia de nueve tipos de compuestos organoclorados en los sedimentos de 19 estaciones de muestreo ubicadas desde la desembocadura del río Grijalva y el río Usumacinta, hasta la 
desembocadura del rio Chumpán, dentro de la Laguna de Términos, lo que incluye la zonas de muestreo del presente estudio, tales como San Pedro, Atasta y Laguna de Términos. Los niveles más altos, de dichos plaguicidas organoclorados, fueron o,p'-DDE $(0.017 \mathrm{mg} / \mathrm{kg})$, seguido de o,p'-DDD $(0.087 \mathrm{mg} / \mathrm{kg})$ y p,p'-DDT $(0.032 \mathrm{mg} / \mathrm{kg})$, detectándose también o,p'-DDT, aldrín, $\alpha$-Clordano, $\gamma-\mathrm{HCH}$, epóxido de Heptacloro y Metoxicloro. Por su parte, las comunidades asentadas en la Península de Yucatán y en el Estado de Quintana Roo producen principalmente cultivos de autoconsumo de maíz y frijol, naranja, limón, coco y pasto, con la tecnología tradicional a la cual se han incorporado el uso de agroquímicos, tanto para el control de plagas, como para aumentar los rendimientos (Hernández Aguilar et al. 2004, INE-ODEPA 2007). Así mismo, en la bahía de Chetumal se realizan actividades agrícolas de cultivos de caña (Cisneros et al. 2011) lo cual podría estar relacionado con la presencia de estos compuestos en el tejido graso del manatí. Adicionalmente, los manatíes que habitan en la bahía de Chetumal, en el Estado de Quintana Roo, podrían estar expuestos a contaminantes que llegan desde la corriente costera del sur del Caribe (Kramer y Kramer 2002)

A pesar de que el HCB no se ha producido comercialmente desde finales de los años setenta, éste se utilizó como fungicida hasta mediados de la década de los 80's, al menos en los EU. Sin embargo, debido a su proveniencia de los procesos industriales y su persistencia, este compuesto se puede encontrar principalmente en los suelos y sedimentos de ríos, lagunas y océanos (ATSDR 2015). También puede encontrarse en el aire, las aguas superficiales y las aguas subterráneas, por lo que logra acumularse a través de la trama trófica y ha sido detectado incluso en la leche materna (ATSDR 2015). A pesar de que el Lindano no ha sido producido en México, éste es importado y utilizado en el país (INE 2004). El Lindano se utilizó como insecticidas en frutas, hortalizas y cultivos forestales, así como en animales, para el combate de ectoparásitos (INE 2004). El Lindano y otros isómeros de $\mathrm{HCH}$, originados por las condiciones ambientales, se han detectado en el aire, en las aguas superficiales y profundas, en sedimento, suelo, peces y otros organismos acuáticos, en animales terrestres, en alimentos y en humanos. (ATSDR 2005), ya que se adsorben fuertemente a suelos con alto contenido de materia orgánica. Sin embargo, la volatilización es una ruta importante de disipación bajo condiciones tropicales y altas temperaturas. La degradación rápida del Lindano ocurre por exposición a la radiación ultravioleta, formando pentaclorociclohexanos y tetraclorociclohexanos. La vida media para la degradación ambiental del Lindano varía de algunos días hasta 3 años dependiendo de varios factores, como el tipo de suelo y el clima (INE 2004). El HCH grado técnico no se utiliza más como insecticida, pero debido a la alta persistencia en el ambiente de algunos isómeros, estos pueden permanecer en el aire durante largos periodos y viajar grandes distancias dependiendo de las condiciones ambientales. Por ello, se han encontrado $\alpha-, \beta-, \gamma-\mathrm{y}$ $\delta$ - $\mathrm{HCH}$ en el suelo, en el aire y en aguas superficiales en sitios de desechos peligrosos, ya que los diferentes isómeros de $\mathrm{HCH}$ pueden estar presentes como vapor o adherirse a partículas pequeñas de tierra y polvo. Las partículas pueden ser removidas del aire por la lluvia o degradadas por otros compuestos que se encuentran en la atmósfera, liberándolo y llevándolo a otros medios como son los cuerpos de agua (ATSDR 2005). El DDT es un insecticida que fue ampliamente utilizado en el pasado para controlar insectos vectores transmisores de enfermedades como la malaria; actualmente se utiliza en pocos países, especialmente africanos, para controlar esta enfermedad. Sin embargo debido a su alta persistencia en el ambiente, es posible encontrarlo aun en la tierra, polvos superficiales, sedimentos y en cuerpos de agua, así como en grasa de animales de vida libre (ATSDR 2002), lo cual sugiere que como resultado de algunas de estas actividades, aún existen cantidades variables en el ambiente, que exponen a los organismos vivos y se acumulan en fase lipídica del cuerpo.

\section{CONCLUSIONES}

El presente estudio aporta evidencia de la presencia de plaguicidas organoclorados en tejido graso de mamíferos acuáticos varados en diferentes regiones del Golfo de México, tanto de la especie T. manatus manatus, así como T. truncatus. En la muestra de grasa de un manatí proveniente de Tabasco se encontró la mayor variedad de compuestos evaluados; sin embargo, en el tejido colectado en Quintana Roo hubo los mayores niveles de $\sum$ DDT. En las toninas los compuestos más abundantes fueron los isómeros de DDT y el metabolito p,p'-DDE con una concentración máxima de $\sum$ DDT de $10.757 \mathrm{mg} / \mathrm{kg}$ en base lipídica. Por tales motivos, es importante mantener un sistema de monitoreo de la presencia de contaminantes ambientales en organismos que habitan en el Área de Protección de Flora y Fauna "Laguna de Términos", ya que se considera la zona de crianza más importante en el sureste del Golfo de 
México para esta especie y otras. Así mismo, deberían realizarse estudios similares en zonas donde habitan diversas especies de mamíferos marinos a lo largo de nuestros litorales.

\section{AGRADECIMIENTOS}

Los autores agradecen el apoyo de la Red Temática de Toxicología de Plaguicidas (CONACyT280045).

\section{REFERENCIAS}

Adams J., Speakman T., Zolman E., Mitchum G., Wirth E., Bossart G.D. y Fair P.A. (2014). The relationship between land use and emerging and legacy contaminants in an Apex predator, the bottlenose dolphin (Tursiops truncatus), from two adjacent estuarine watersheds. Environ. Res. 135, 346-353.

DOI: 10.1016/j.envres.2014.08.037

Aguilar A., Borrell A. y Reijnders P.J.H. (2002). Geographical and temporal variation in levels of organochlorine contaminants in marine mammals. Mar. Environ. Res. 53 (5), 425-452.

DOI: $10.1016 / \mathrm{S} 0141-1136(01) 00128-3$

Aguirre A.A. y Tabor G.M. (2004). Introduction: Marine vertebrates as sentinels of marine ecosystem health. Eco. Health. 1 (3), 236-238.

DOI: $10.1007 / \mathrm{s} 10393-004-0091-9$

Albert L.A. y Loera Gallardo R. (2005). Química y ecotoxicología de los insecticidas. En: Golfo de México. Contaminación e impacto ambiental: Diagnóstico y tendencias. (A.V. Botello, J. Rendón-von Osten, G. GoldBouchot y C. Agraz-Hernández, Eds.) Univ. Autón. de Campeche, Univ. Nal. Autón. de México, Instituto Nacional de Ecología. Campeche, México, pp. 177-190.

ATSDR (2002). Toxicological Profile for DDT, DDE, and DDD. U.S. Department of Health and Human Services, Agency for Toxic Substances and Disease Registry. Guía técnica. Atlanta, EUA, 497 pp.

ATSDR (2005). Toxicological Profile for alpha-, beta-, gamma-, and delta-hexachlorocyclohexane. U.S. Department of Health and Human Services, Agency for Toxic Substances and Disease Registry. Guía técnica. Atlanta, EUA, 377 pp.

ATSDR (2015). Toxicological Profile for Hexachlorobenzene. U.S. Department of Health and Human Services, Agency for Toxic Substances and Disease Registry. Guía técnica. Atlanta, EUA, 432 pp.

Benítez J.A. y Bárcenas C. (1996). Patrones de uso de los plaguicidas en la zona costera del Golfo de México.
En: Golfo de México, contaminación e impacto ambiental: Diagnostico y tendencias (A.V. Botello, J.L. Rojas Galaviz, J.A. Benítez y D. Zárate-Lomelí, Eds.) EPOMEX Serie Científica 5. Universidad Autónoma de Campeche. Campeche, México, pp. 155-167.

Brown A.W.A. (1978). Ecology of pesticides. John Wiley \& Sons. New York, EUA. 525 pp.

Cisneros E., Rivas A., Díaz, J. y Castanedo V. (2011). Tratamiento de las aguas residuales del ingenio Casasano en un humedal de flujo intermitente. IMTASEMARNAT, [en línea]. https://www.agua.org.mx/ wp-content/uploads/2011/07/tratamientoaguasresidualesdelingeniocasasano.pdf 19/01/2017

Colborn T. y Clement C. (1992). Chemically induced alterations in sexual and functional development: The wildlife/human connection. Advances in modern environmental toxicology. Princeton Scientific Pub. Co., Princeton, NJ., USA. 403 pp.

Corsolini S., Focardi S., Kannan K., Tanabe S., Borrell A. y Tatsukawab R. (1995). Congener profile and toxicity assessment of polychlorinated biphenyls in dolphins, sharks and tuna collected from Italian coastal waters. Mar. Environ. Res. 40 (1), 33-53.

DOI: $10.1016 / 0141-1136(94) 00003-8$

Delgado-Estrella A. (2002). Comparación de parámetros poblacionales de las toninas, Tursiops truncatus, en la región sureste del Golfo de México (Estados de Tabasco, Campeche, Yucatán y Quintana Roo). Tesis Doctoral. Facultad de Ciencias, Universidad Nacional Autónoma de México. Ciudad de México, México. 160 pp.

Hansen L.J., Schwacke L.H., Mitchum G.B., Hohn A.A., Wells, R.S., Zolman, E.S. y Fair P.A. (2004). Geographic variation in polychorinated biphenyl and organochlorine pesticide concentrations in the blubber of bottlenose dolphins from the US Atlantic coast. Sci. Total Environ. 319 (1-3), 147-172.

DOI: $10.1016 / \mathrm{S} 0048-9697(03) 00371-1$

Haynes D., Carter S., Gaus, C., Muller, J. y Dennison W. (2005). Organochlorine and heavy metal concentrations in blubber and liver tissue collected from Queensland (Australia) dugong (Dugong dugon). Mar. Pollut. Bull. 51 (1-4), 361-369.

DOI: $10.1016 /$ j.marpolbul.2004.10.020

Hernández Aguilar M.L., de Fuentes G., Córdoba A. y Ordóñez J. (2004). Distribución territorial de la producción agropecuaria en la Península de Yucatán hacia finales del siglo XX. Centro de Investigación y Estudios Avanzados del IPN-Unidad Mérida, Universidad Nacional Autónoma de México, Instituto de Geografía: Programa de Pequeñas Donaciones del Fondo para el Medio Ambiente Mundial. Mérida, México. 40 pp.

INE (2004). El lindano en México. Instituto Nacional de Ecología. Informe, México D.F., México. 67 pp. 
INE-ODEPA (2007). Censo agropecuario y forestal 2007. Ministerio de Agricultura; Instituto Nacional de Estadística (INE), Oficina de Estudios y Políticas Agrarias (ODEPA) Chile. [en línea] http://www.ine. cl/canales/chile_estadistico/censos_agropecuarios/ censo_agropecuario_07_comunas.php 19/01/2017

Jackson T.F. y Halbert F.L. (1974). A toxic syndrome associated with the feeding of polybrominated biphenylcontaminated protein concentrate to dairy cattle. J. Am. Vet. Med. Assoc. 165 (5), 437-439. PMID: 4425399

King C.A. (1987). Organochlorines in bottlenose dolphins (Tursiops truncatus) and pygmy sperm whales (Kogia breviceps) from southeastern Florida. Tesis de Maestría. University of Miami. Coral Gables, FL. EUA. 92 pp.

Kramer P.A. y Kramer P.R. (2002). Ecoregional Conservation Planning for the Caribbean Reef (MACR). World Wildlife Fund (WWF). Washington, D.C., EUA. 140 pp.

Kuehl D.W. y Haebler R. (1995). Organochlorine, organobromine, metal, and selenium residues in bottlenose dolphins (Tursiops truncatus) collected during an unusual mortality event in the Gulf of Mexico, 1990. Arch. Environ. Contam. Toxicol. 28 (4), 494-499.

DOI: 10.1007/BF00211632

Lahvis G.P., Wells R. S., Kuehl D.W., Stewart J.L., Rhinehart H.L. y Via C.S. (1995). Decreased lymphocyte responses in free-ranging bottlenose dolphins (Tursiops truncatus) are associated with increased concentrations of PCBs and DDT in peripheral blood. Environ. Health Perspect. 103 (4), 67-72. PMC1519269

Marsili L. y Focardi S. (1997). Chlorinated hydrocarbon (HCB, DDTs and PCBs) levels in cetaceans stranded along the Italian coasts: an overview. Environ. Monit. Assess. 45 (2), 129-180.

DOI: $10.1023 / \mathrm{A}: 1005786627533$

Moore S.E. (2008). Marine mammals as ecosystem sentinels. J. Mammal. 89 (3), 534-540.

DOI: 10.1644/07-MAMM-S-312R1.1

Ortega-Argueta A. y Morales-Vela B. (2005). Status and conservation of the manatee (Trichechus manatus) in Mexico. Memorias. IX th International Mammalogical Congress. Sapporo, Japón. 31 de julio al 5 de agosto, 2005.

PNUMA (2003). GEO América Latina y el Caribe, perspectivas del medioambiente 2003. Oficina Regional para América Latina y el Caribe, Programa de las Naciones Unidas para el Medio Ambiente. Informe regional. Costa Rica, $281 \mathrm{pp}$.

PNUMA (2011). Convenio de Estocolmo sobre contaminantes orgánicos persistentes (COP). Secretaría del Convenio de Estocolmo sobre Contaminantes Orgánicos Persistentes, Programa de las Naciones Unidas para el Medio Ambiente. Textos y anexos. Genova, Suiza. 60 pp.
Reddy M.L., Dierauf L.A. y Gulland F.M.D. (2001). Marine mammals as sentinels of ocean health. En: CRC Handbook of marine mammal medicine: Health, disease, and rehabilitation. (L.A. Dierauf y F.M.D. Gulland Eds.) CRC Press, Taylor \& Francis Group. Boca Raton, FL. EUA. 3-13.

Rendón-von Osten J., Memije Canepa M. y Ek Moo N.A. (2005). Plaguicidas orgánicos persistentes (POPs) en sedimentos de la costa sur de Campeche, México. En: Golfo de México contaminación e impacto ambiental: Diagnóstico y tendencias (A.V. Botello, J. Rendón-von Osten, G. Gold-Bouchot y C. AgrazHernández Eds.) Univ. Autón. de Campeche, Univ. Autón. de Campeche, Univ. Nal. Autón. de México, Instituto Nacional de Ecología. Campeche, México, pp. 249-260.

Rolland R., Michael G. y Peterson R.E. (1997). Chemically induced alterations in functional development and reproduction of fishes. SETAC Press. Pensacola, Fl., EUA. 224 pp.

Salata G.G., Wade T. L., Sericano J.L., Davis J.W. y Brooks J.M. (1995). Analysis of Gulf of Mexico bottlenose dolphins for organochorine pesticides and PCBS. Environ. Pollut. 88 (2), 167-175.

DOI: 10.1016/0269-7491(95)91441-M

SEMARNAT (2013). Convenio de Estocolmo. Secretaría de Medio Ambiente y Recursos Naturales. [en línea]. http://www.semarnat.gob.mx/temas/agenda-internacional/convenio-de-estocolmo 19/01/2017

SEMARNAT (2014). Protocolo de atención para varamientos de mamíferos marinos. Secretaría de Medio Ambiente y Recursos Naturales. Protocolo. Distrito Federal, México. 28 pp.

Stahl R.G. (1997). Can mammalian and non-mammalian "sentinel species"; data be used to evaluate the human health implications of environmental contaminants? Hum. Ecol. Risk. Assess. 3 (3) 329-335.

DOI: $10.1080 / 10807039709383689$

Van Der Schalie W.H., Gardner Jr. H.S., Bantle J.A., De Rosa C.T., Finch R.A., Reif J.S., Reuter R.H., Backer L.C., Burger J., Folmar L.C. y Stokes W.S. (1999). Animals as sentinels of human health hazards of environmental chemicals. Environ. Health Perspect. 107 (4), 309-315. PMC1566523

Vetter W., Scholz E., Gaus C., Müller J.F. y Haynes D.W. (2001). Anthropogenic and natural organohalogen compounds in blubber of dolphins and dugongs (Dugong dugon) from northeastern Australia. Arch. Environ. Contam. Toxicol. 41 (2), 221-231.

DOI: $10.1007 / \mathrm{s} 002440010241$

Wagemann R. y Muir D.C.G. (1984). Concentrations of heavy metals and organochlorines in marine mammals of northern waters overview and evaluation. Canadian 
Department of Fisheries and Oceans. Reporte Técnico. Winnipeg, Canada, $107 \mathrm{pp}$.

Waliszewski S.M. y Szymczynski G.A. (1982). Simple, low-cost method for determination of selected chlorinated pesticides in fat samples. J. Assoc. Off. Anal. Chem, 65 (3), 677-679. PMID: 7096250

Waliszewski S.M., Infanzon R.M. y Hart M.M. (2003). Differences in persistent organochlorine pesticides concentration between breast adipose tissue and blood serum. Bull. Environ. Contam. Toxicol. 70 (5), 920-926.

DOI: $10.1007 / \mathrm{s} 00128-003-0070-9$

Wilson R. M., Kucklick J. R., Balmer B. C., Wells R. S., Chanton J. P. y Nowacek D. P. (2012). Spatial distribution of bottlenose dolphins (Tursiops truncatus) inferred from stable isotopes and priority organic pollutants. Sci. Total Environ. 425, 223-230.

DOI: 10.1016/j.scitotenv.2012.02.030 